\title{
Split donor centers and split excitons in a semiconductor heterostructure
}

\author{
Z. S. Gribnikov a) and G. I. Haddad \\ Solid State Electronics Laboratory, Department of Electrical Engineering and Computer Science, University \\ of Michigan, Ann Arbor, Michigan 48109
}

(Received 2 June 2005; accepted 29 August 2005; published online 14 October 2005)

\begin{abstract}
The first subject considered in the article is a donor center embedded in a thin heterostructural barrier separating a semiconductor medium into two halves. As a result of the small thickness of this barrier, the wave function of an electron bound by the donor center shifts almost completely into both halves of the surrounding semiconductor medium. The ground and first excited electron states of such a donor center are separated from each other by a narrow energy gap determined by the symmetric-antisymmetric tunnel splitting. Such structures can be implemented in both $\mathrm{GaAs} / \mathrm{Al}_{X} \mathrm{Ga}_{1-X} \mathrm{As}$ and $\mathrm{Si} / \mathrm{Ge}_{X} \mathrm{Si}_{1-X}$ material systems. The second considered subject is an exciton formed in analogous heterostructures when the staggered band alignment takes place between the heterobarrier and semiconductor medium. As a result of such band alignment, the hole participating in the exciton creation is located in the formed quantum well and the electron, which is the hole's opponent, is separated into halves (on different sides of the quantum well) as before. Unlike the donor center, the exciton can be shifted and localized in arbitrary positions along the staggered "barrier-well" boundary by inhomogeneous electric fields of external controlling gates. (C) 2005 American Institute of Physics. [DOI: 10.1063/1.2084317]
\end{abstract}

\section{INTRODUCTION}

Two-level quantum nanostructures manipulated by small charged metallic gates are interesting as active elements of the modern nanoelectronics and quantum information sciences. Two two-level quantum subjects are considered theoretically in this article. The first is a donor center, located in a thin heterostructural barrier separating a semiconductor medium into two halves (Fig. 1). The wave function of the electron bound by this donor center is also split into two halves placed on different sides of the heterobarrier and connected weakly with each other by tunneling through the barrier. As a result of this weak connection, we obtain a twolevel quantum system with a symmetric-antisymmetric (SAS) tunnel splitting of an electron ground state (see Secs. II and III).

The second subject we consider is a specific exciton formed in the analogous heterostructure in the case when the type-II (staggered) band alignment takes place (Sec. IV). We assume that the same heterostructural barrier as above appears in the semiconductor conduction band and separates the electron wave function of the exciton into two halves. Simultaneously, a quantum well is formed in the valence band and localizes the hole part of the exciton wave function. This localized hole serves as the above-described donor center. In this case the ground exciton state also experiences a tunnel S-AS splitting. The considered specific exciton exists only around the heterobarrier plane and occupies an arbitrary free position along this plane. It can be shifted by inhomogeneous electric fields, which pull the exciton into the maximum field position.

The consideration of the above-mentioned problems is fulfilled on the basis of a simplified model. The heterobarrier

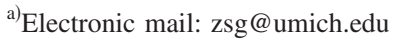

potential is modeled as a $\delta$-function potential in the donor center case and as a combination of a $\delta$-function barrier with a "quasi- $\delta$-function" quantum well in the exciton case. Such a simplified approach needs a minimal number of the parameters describing the heterostructure (but, of course, cannot be complete). The semiconductor medium, in which the electron wave function is basically located, is described below by both an isotropic dispersion relation with a single effective mass (Sec. II) and an anisotropic dispersion relation with two effective masses (Sec. III). The latter case is related to the case of $\mathrm{Ge}_{X} \mathrm{Si}_{1-X} / \mathrm{Si}$ material systems when tensilestressed Si layers serve as the above-mentioned semiconductor medium.

\section{SPLIT DONOR CENTER IN THE ISOTROPIC CASE}

We consider a thin heterostructural barrier grown in an undoped semiconductor and containing a shallow donor center (DC). The suitable illustrative example is an $\mathrm{Al}_{X} \mathrm{Ga}_{1-X} \mathrm{As}$ barrier in a GaAs medium. Shallow donors with a low concentration $\left(\leqslant 10^{11} \mathrm{~cm}^{-2}\right)$ are assumed in the barrier. In this

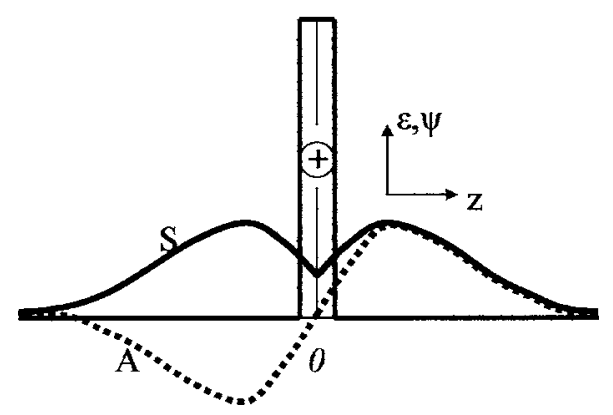

FIG. 1. Qualitative sketch of an electron wave function $\Psi(0, z)$ of the ground $(S)$ and the first excited $(A)$ quantization states for a split donor center in a thin heterostructural potential barrier. 
case, the average distance $r_{1}$ between the donors in the barrier $(\geqslant 30 \mathrm{~nm})$ exceeds substantially the effective Bohr radius $r_{B}=\hbar^{2} \kappa_{D} / e^{2} m$ for an electron in the GaAs medium $(\sim 8 \mathrm{~nm})$. Here $\kappa_{D}$ and $m$ are the dielectric constant and $\Gamma$-electron effective mass for GaAs, respectively. The abovementioned Bohr radius is assumed to be much larger than the $\mathrm{Al}_{X} \mathrm{Ga}_{1-X} \mathrm{As}$ barrier thickness, which is hypothetically equal to several lattice constants: $w \leqslant 1 \mathrm{~nm}$. So we have

$$
r_{1} \gg r_{B} \gg w .
$$

Since impurity (donor) centers are localized in the comparatively high heterobarrier, hypothetical thermodynamicequilibrium electrons will leave the donor neighborhood (and the barrier in general) and step down into the surrounding GaAs medium. In the high-temperature case, the donor electron becomes one of the free electrons in GaAs, but at sufficiently low temperatures such an electron is localized around its donor center on both sides of the heterobarrier and forms the distinctive H-like atom, which is split into two halves by the heterobarrier. Electron states in such a split donor center (SDC) are subjects of our particular interest. We continue our consideration using a simplified model: we replace the splitting heterobarrier having its real complicated potential, which can be described by means of numerous parameters, by a simple $\delta$ potential. The resulting electron Hamiltonian is

$$
\hat{H}=\hat{p}^{2} / 2 m-e^{2} / \kappa_{D} r+U_{0} \delta(z),
$$

where $\hat{p}=-i \hbar \partial / \partial \mathbf{r}, r=\left(r_{\perp}^{2}+z^{2}\right)^{1 / 2}, r_{\perp}^{2}=x^{2}+y^{2}$, the $z$ axis is directed along the normal to the heterobarrier, the $x$ and $y$ axes are in its plane, and the introduced coefficient $U_{0}$ serves as the single parameter of the splitting barrier.

The splitting $\delta$ potential changes the electron spectrum of the SDC in comparison with the unsplit DC. The $\delta$ potential excludes all the $s$ states of the spherically symmetric $\mathrm{H}$-like center problem ${ }^{1}$ and first of all the ground $1 s$ state. All the other energy levels of the DC connected with $p, d, f$, etc., states exist also in the SDC, but multiplicities of their degeneracy are noticeably lowered. The last component on the right side of Eq. (2) cannot affect the DC electron states, for which the electron wave functions $\Psi(x, y, z=0)=0$ (that is, for all the asymmetric states in relation to the plane $z=0)$. These states include all the DC states with nonzero moments of momentum directed arbitrarily along the plane $z=0$ and with nonzero magnetic quantum numbers $|m| \neq 0$. In this latter case, we can always construct states with real factors ${ }^{1}$ $\sin m \varphi$ or $\cos m \varphi$ that are equal to 0 in the plane $z=0$ (where $\varphi$ is an angle in the plane, which is normal to the moment of momentum). We should also add to the above-mentioned states all the states with moments of momentum directed along the $z$ axis, for which $l-|m|$ is odd: $l-|m|=1,3,5$, and so on (where $l$ is an orbital quantum number for the DC). Note again that all the above-listed states are antisymmetric on $z$,

$$
\Psi(x, y, z)=-\Psi(x, y,-z),
$$

and belong to both the complete Hamiltonian (2) for the SDC and the Hamiltonian (2) without the last component on the right side, which describes the usual (unsplit) DC.
Let us imagine that the coefficient $U_{0}$ in Eq. (2) is equal to $\infty$. Then, each of the above-listed antisymmetric solutions (3) also generates the additional symmetric solution of Eq. (2), which can be constructed from the solution for $z>0$ by an even (symmetric) extension in the region $z<0, \Psi(x, y, z)=\Psi(x, y,-z)$. We have for such solutions $\Psi(x, y, 0)=0$ as before, but we have a discontinuous first derivative at $z=0, \partial \Psi(x, y, z) /\left.\partial z\right|_{z>0}=-\partial \Psi(x, y, z) /\left.\partial z\right|_{z<0}$. Note that these symmetric solutions with discontinuous derivatives $\partial \Psi / \partial z$ at $z=0$ do not represent any DC states. They correspond only to SDC states for $U_{0}=\infty$. Similar symmetric fundamental states for the Hamiltonian (2) exist also for large but finite values of $U_{0}$. They do not coincide with absolute values of the antisymmetric DC and SDC states being different from them near the plane $z=0$ where $\Psi(x, y, 0)$ $\neq 0$ for these symmetric SDC states. The approximate value of $\Psi(x, y, 0)$ can be obtained directly with the help of the Hamiltonian (2) in the form

$$
\Psi(x, y, 0) \cong\left(\hbar^{2} / m U_{0}\right) \partial \Psi^{(0)}(x, y, z) /\left.\partial z\right|_{z=0},
$$

where $\Psi^{(0)}(x, y, z)$ is the wave function of the corresponding antisymmetric state.

The lowest DC (and SDC) antisymmetric state is a $2 p$ state with the energy

$$
E_{2}^{(\mathrm{AS})}=-\varepsilon_{0} / 8
$$

where $\varepsilon_{0}=m e^{4} / \hbar^{2} \kappa_{D}^{2}$ and with the wave function

$$
\Psi(x, y, z)=\Psi\left(\rho_{\perp}, \zeta\right)=C \zeta \exp (-\rho / 2),
$$

where $\zeta=z / r_{B}, \rho=r / r_{B}, \rho_{\perp}=r_{\perp} / r_{B}$, and $\rho^{2}=\zeta^{2}+\rho_{\perp}^{2}$.

In order to take into account the energy correction connected with a finite value of $U_{0}$ and a nonzero value of $\Psi\left(\rho_{\perp}, 0\right)$ we use the variation principle ${ }^{1,2}$

$$
\varepsilon \leqslant \int \Psi^{*} \hat{H} \Psi d \tau / \int|\Psi|^{2} d \tau .
$$

Let us select a trial function in the form

$$
\Psi=C(|\zeta|+Z) \exp (-\alpha \rho),
$$

where $Z$ and $\alpha$ are variation parameters. This trial function differs from the well-known wave function (6) just by these variation parameters: $Z$ should not be 0 because of some electron penetration into the barrier, and $\alpha$ cannot be equal to $1 / 2$. The following calculations confirm that a small difference between $\alpha$ and $1 / 2$ does not lead to a perceptible correction. We obtain the right side of Eq. (7) in the form

$$
E_{1}^{(S)}=E(\alpha, Z)=\left(\varepsilon_{0} / 2\right)\left[\alpha^{2}-\alpha f(X)\right]+\varepsilon_{1} g(X),
$$

with $f(X)=\left(1+X+X^{2} / 2\right) /\left(1+3 X / 4+X^{2} / 4\right), \quad g(X)=X^{2} /(1$ $\left.+3 X / 4+X^{2} / 4\right), X=2 \alpha Z$, and $\varepsilon_{1}=U_{0} / 8 r_{B}$. In the interesting case of the high heterobarrier when $\varepsilon_{1} \gg \varepsilon_{0}$ (that is, $U_{0}$ $\left.\gg 8 e^{2} / \kappa_{D}\right)$, we obtain $X \cong \varepsilon_{0} / 32 \varepsilon_{1}=\varepsilon_{0} r_{B} / 4 U_{0} \ll 1, \alpha=1 / 2$ $+\varepsilon_{0} / 256 \varepsilon_{1}=1 / 2+X / 8$, and

$$
E_{1}^{(S)}=-\left(\varepsilon_{0} / 8\right)\left(1+\varepsilon_{0} r_{B} / 16 U_{0}\right)=-E_{2}^{(\mathrm{AS})}(1+X / 4) .
$$

We have also evaluated $E$ with the help of a somewhat different trial function, $\Psi=C\left[|\zeta| \exp (-\rho / 2)+Z^{\prime} \exp (-\rho)\right]$, compiled as a sum of the wave functions of the DC ground 
and first excited states with the single variation parameter $Z^{\prime}$. The optimal value of this parameter is $Z^{\prime} \cong 8 \varepsilon_{0} r_{B} / 9 U_{0}$ and we obtain $E_{1}^{(S)}=-\left(\varepsilon_{0} / 8\right)\left[1+(8 / 81) \varepsilon_{0} r_{B} / U_{0}\right]=-\left(\varepsilon_{0} / 8\right)(1$ $+16 X / 81)$, that is, somewhat worse than in Eq. (10).

The model heterobarrier, which splits the ground H-like state of the DC, draws closer its even ground state and odd first excited state and transforms them into a two-level system with the distinctive S-AS level splitting. The structure of this splitting does not contain the typical exponential factor taking place in the case of thicker and lower barriers. Such a factor could not be described by the simple $\delta$-function model. ${ }^{3}$

\section{DONOR CENTER IN THE ANISOTROPIC SILICON CASE}

The $\mathrm{Al}_{X} \mathrm{Ga}_{1-X} \mathrm{As}$ barrier in the GaAs medium discussed above as the simplest example may not be the most attractive from the viewpoint of applications. Now there exists great interest ${ }^{4,5}$ in $\mathrm{Si}_{1-X} \mathrm{Ge}_{X} / \mathrm{Si}$ heterostructures grown as a rule on virtual $\mathrm{Si}_{1-X} \mathrm{Ge}_{X}$ substrates with $X=0.3-0.4$ (see Ref. 6 as a review). In this case, tensile-stressed $\mathrm{Si}$ layers, which are stretched on the above-mentioned virtual substrates, cannot be infinitely thick, but can be sufficiently thick. For example, perfect Si layers with $26 \mathrm{~nm}$ thicknesses grown on the virtual $\mathrm{Si}_{0.7} \mathrm{Ge}_{0.3}$ substrate are described in Refs. 7 and 8. Being separated by a thin $\mathrm{Si}_{1-X} \mathrm{Ge}_{X}$ barrier (at $X=0.3-0.4$ ) such layers are sufficiently vast to accommodate undistorted wave functions of the SDC placed in this splitting barrier. The above-indicated stretching of the Si medium in the $x y$ plane with its compression along the $z$ axis normal to the barrier induces a noticeable energy lowering of the two equivalent electron valleys directed along the same $z$ axis $\left(\Delta_{2}\right.$ valleys) in comparison with the rest of the four valleys $\left(\Delta_{4}\right.$ valleys). This lowering lifts the multivalley problem, but the electron spectrum continues to be anisotropic. The corresponding Hamiltonian replacing the one in Eq. (2) is

$$
\hat{H}=\hat{p}_{\perp}^{2} / 2 m_{\perp}+\hat{p}_{z}^{2} / 2 m_{Z}-e^{2} / \kappa_{D} r+U_{0} \delta(z),
$$

where $m_{\perp}=m_{X}=m_{Y}$ is a small transverse mass, $m_{Z}$ is a large longitudinal mass, $\hat{p}_{\perp}=-i \hbar \partial / \partial \mathbf{r}_{\perp}$, and $\hat{p}_{Z}=-i \hbar \partial / \partial z$. We have neglected a small anisotropy of the dielectric constant $\kappa_{D}$ in the Si medium. In contrast to the Hamiltonian (2), the Hamiltonian (11) allows us to obtain accurate wave functions neither for the case $U_{0}=0$ nor for the case $U_{0}=\infty$. By tradition, we can use the variation procedure based on the trial function in the form ${ }^{9-11}$

$$
\Psi\left(\zeta, \rho_{\perp}\right)=C_{1} \exp \left(-\sqrt{\alpha_{1}^{2} \zeta^{2}+\beta_{1}^{2} \rho_{\perp}^{2}}\right)
$$

for the ground $(1 s)$ state and on the trial function in the form $^{10,12}$

$$
\Psi\left(\zeta, \rho_{\perp}\right)=C \zeta \exp \left(-\sqrt{\alpha^{2} \zeta^{2}+\beta^{2} \rho_{\perp}^{2}}\right)
$$

for the first excited $\left(2 p_{0}\right)$ state. This state is antisymmetric in relation to the plane $\zeta=0$. As before, we have introduced $\zeta=z / r_{B}$ and $\rho_{\perp}=r_{\perp} / r_{B}$ on the right sides of Eqs. (12) and (13) where $r_{B}=\hbar^{2} \kappa_{D} / e^{2} m$ and $m=m_{z}^{1 / 3} m_{\perp}^{2 / 3}$. Below we are interested in the case when $m_{Z}>m_{\perp}$. In the specific case of
Si, we select $m_{Z}=0.92 m_{0}$ and $m_{\perp}=0.19 m_{0}$. Then we have $m \cong 0.32 m_{0}$ and $\gamma=m_{\perp} / m_{Z} \cong 0.206$.

For the electron wave function of the considered SDC placed in the $\delta$-shaped heterobarrier and described by the model Hamiltonian (11), we can select as a trial function [analogously to the function (8)]

$$
\Psi=C(|\zeta|+Z) \exp \left(-\sqrt{\alpha^{2} \zeta^{2}+\beta^{2} \rho_{\perp}^{2}}\right) .
$$

In the function (14) we have three variation parameters $\alpha, \beta$, and $Z$ instead of two parameters for an isotropic valley when $\alpha=\beta$. The right-hand side of Eq. (7) in the considered anisotropic case can be presented by three components,

$$
\begin{aligned}
E= & E(a, p, Z)=\left(\varepsilon_{0} / 2\right)\left[\alpha^{2} \Phi_{1}(p, Z)-\alpha \Phi_{2}(p, Z)\right. \\
& +\alpha g(Z) / \lambda],
\end{aligned}
$$

where the first of them originated by the first two components of the Hamiltonian (11) represents an electron kinetic energy, the second one represents an electron-donor interaction, and the last component expresses an interaction between an electron and the $\delta$-barrier potential. In Eq. (15) we have

$$
\begin{aligned}
\begin{aligned}
\Phi_{1}(p, Z)= & \gamma^{2 / 3}+\left[\Phi_{10}(p)-\gamma^{2 / 3}\right] r(Z) / q(Z), \\
\Phi_{2}(p, Z)= & \left\{\Phi_{20}(p)+Z\left[\Phi_{21}(p)+(3 / 4) \Phi_{20}(p)\right]\right. \\
& \left.+Z^{2} / 2\right\} / q(Z),
\end{aligned} \\
\begin{aligned}
& \Phi_{10}(p)=(3 / 5) \gamma^{2 / 3}+(2 / 5) \gamma^{-1 / 3} /\left(1+p^{2}\right), \\
& \Phi_{20}(p)=(3 / 2)\left[\left(p^{2}+1\right) \arctan p-p\right] / p^{3},
\end{aligned} \\
\begin{aligned}
\Phi_{21}(p)=2\left(\sqrt{p^{2}+1}-1\right) / p^{2}-(3 / 4) \Phi_{20}(p), \\
\lambda=\varepsilon_{0} / 4 \varepsilon_{1}=2 \varepsilon_{0} r_{B} / U_{0} \ll 1, \varepsilon_{0}=m e^{4} / \hbar^{2} \kappa_{D}^{2},
\end{aligned} \\
\varepsilon_{1}=(1 / 8) U_{0} / r_{B}, \quad g(Z)=Z^{2} / q(Z), \\
r(Z)=1+(15 / 16) Z+(5 / 12) Z^{2}, \\
q(Z)=1+(3 / 4) Z+(1 / 4) Z^{2}, \\
p^{2}=\left(\alpha^{2} / \beta^{2}\right)-1 .
\end{aligned}
$$

Below, we use the parameter $p$ introduced by Eq. (15e) as the variation parameter (instead of the parameter $\beta$ and together with the parameters $\alpha$ and $Z$ ). The optimal values of $\alpha, p$, and $Z$ are defined by the following equations:

$$
\begin{aligned}
& 2 \alpha \Phi_{1}(p, Z)-\Phi_{2}(p, Z)+(1 / \lambda) g(Z)=0, \\
& \alpha \partial \Phi_{1} / \partial p-\partial \Phi_{2} / \partial p=0, \\
& \alpha \partial \Phi_{1} / \partial Z-\partial \Phi_{2} / \partial Z+(1 / \lambda) d g / d Z=0 .
\end{aligned}
$$

The parameter $\lambda \ll 1$ introduced by Eq. (15d) can be considered as the small parameter of the problem allowing us to obtain our solutions in the form 
$\alpha=\alpha_{0}+\alpha_{1} \lambda+\cdots, \quad p=p_{0}+p_{1} \lambda+\cdots, \quad Z=Z_{1} \lambda+\cdots$.

It is evident that the case $\lambda=0$ corresponds to a solution of the problem for the $2 p$ state without the separating barrier or the same problem for the ground state at $U_{0}=\infty$. Taking into account components linear in $\lambda$ allows us to calculate the correction, which is analogous to the one described by Eq. (10) in the isotropic case.

Realization of the above-described procedure leads to the equations

$$
\alpha_{0}=(1 / 2) \Phi_{20}\left(p_{0}\right) / \Phi_{10}\left(p_{0}\right)=\Phi_{20}^{\prime}\left(p_{0}\right) / \Phi_{10}^{\prime}\left(p_{0}\right),
$$

where $\Phi_{10,20}^{\prime}(p)=d \Phi_{10,20} / d p$. Equations (20) allow us to obtain in explicit form

$$
\gamma=(2 / 3) \frac{2 p_{0}^{3}+3 p_{0}-3\left(1+p_{0}^{2}\right) \arctan p_{0}}{\left(1+p_{0}^{2}\right)^{2}\left[\left(3+p_{0}^{2}\right) \arctan p_{0}-3 p_{0}\right]}
$$

and determine $p_{0}(\gamma)$. We can see that $p_{0}(1)=0, p_{0}(0)=\infty$, and in the $\mathrm{Si}$ case with $\gamma \cong 0.206$ we have $p_{0}(0.206)$ $\cong 1.325$. We can obtain from the same Eqs. (20)

$$
\alpha_{0}=\frac{15 \gamma^{1 / 3}\left(1+p_{0}^{2}\right)\left[\left(1+p_{0}^{2}\right) \arctan p_{0}-p_{0}\right]}{4 p_{0}^{3}\left[3 \gamma\left(1+p_{0}^{2}\right)+2\right]}
$$

and then $\beta_{0}=\alpha_{0} /\left(1+p_{0}^{2}\right)^{1 / 2}$. In the $\mathrm{Si}$ case we have $\alpha_{0}$ $\cong 0.865$ and $\beta_{0} \cong 0.521$.

The function $Z_{1}\left(p_{0}\right)$ obtained from Eq. (18) can be presented in the form

$$
Z_{1}\left(p_{0}\right)=-(1 / 2)\left[\alpha_{0} \Phi_{11}\left(p_{0}\right)-\Phi_{21}\left(p_{0}\right)\right],
$$

with $\Phi_{11}(p)=(3 / 40)\left[\gamma^{1 / 3} /\left(1+p^{2}\right)-\gamma^{2 / 3}\right]$; the expression for $\Phi_{21}(p)$ is presented above by Eq. (15c). Taking into account the approximate formulas (19), we can rewrite Eq. (15) in the form

$$
E \cong E_{0}\left(p_{0}\right)+\lambda E_{1}\left(p_{0}\right) \mathrm{Z}_{1}\left(p_{0}\right),
$$

where $E_{0}\left(p_{0}\right)=\left(\varepsilon_{0} \alpha_{0} / 2\right)\left[\alpha_{0} \Phi_{10}\left(p_{0}\right)-\Phi_{20}\left(p_{0}\right)\right]$ and $E_{1}\left(p_{0}\right)$ $=\left(\varepsilon_{0} \alpha_{0} / 4\right)\left[\alpha_{0} \Phi_{11}\left(p_{0}\right)-\Phi_{21}\left(p_{0}\right)\right]$. As a result, we can obtain

$$
\begin{aligned}
E \cong & \left(\varepsilon_{0} \alpha_{0} / 2\right)\left\{\alpha_{0} \Phi_{10}\left(p_{0}\right)-\Phi_{20}\left(p_{0}\right)-(\lambda / 4)\left[\alpha_{0} \Phi_{11}\left(p_{0}\right)\right.\right. \\
& \left.\left.-\Phi_{21}\left(p_{0}\right)\right]^{2}\right\} .
\end{aligned}
$$

In the $\mathrm{Si}$ case $\Phi_{10}\left(p_{0}\right) \cong 0.455, \quad \Phi_{20}\left(p_{0}\right) \cong 0.788$, $\Phi_{11}\left(p_{0}\right) \cong 0.0199$, and $\Phi_{21}\left(p_{0}\right) \cong 0.161$, thus we have $E \approx-\left(\varepsilon_{0} / 2\right)(0.341+0.00447 \lambda)$. Let us compare this result with the analogous formula (10) that was derived for the isotropic dispersion relation and presented in the form $E \approx$ $-\left(\varepsilon_{0} / 2\right)(0.25+0.0156 \lambda)$. We can see that the effect of the splitting heterobarrier in the Si case is substantially smaller than in the isotropic case (for the same values of $\varepsilon_{0}$ and $\lambda$ !). This fact can be explained by the large longitudinal effective mass $m_{Z}$ in the Si case.

\section{SPLIT EXCITON}

The two-level systems appearing as a result of S-AS splitting of the ground electron state in a donor center embedded in a realistic thin heterobarrier can be noticeably different from the above-considered prototype based on the

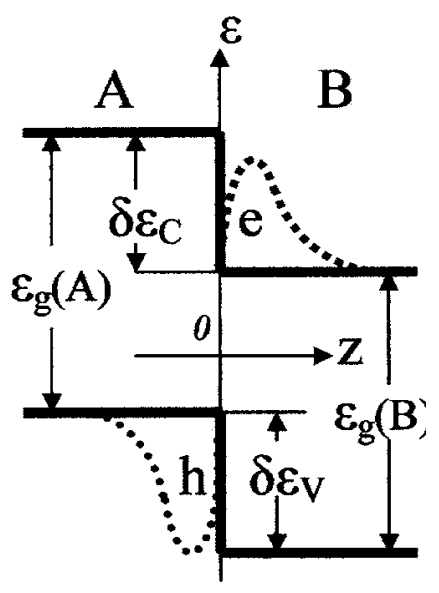

FIG. 2. Model of an interface exciton in the case of a heterojunction with a staggered band alignment.

$\delta$-shaped potential model. First, real donors embedded in a finite-thickness barrier have some spread in the $z$ coordinate and this spread could destroy the suggested precise symmetry and antisymmetry. Second, this finite-thickness thin heterobarrier can be insufficiently symmetric per se. Both of these disadvantages could be eliminated by a correcting electric field of an outer controlling miniature gate, but there appears a problem of the space alignment of this very small gate with the donor center that is not very simple. These problems could be simplified if we replace the donor center localized in the heterobarrier by a hole localized in the same layer. This means that we should turn the donor center with a split electron wave function into a split exciton. The latter could result only if our heterostructure containing a thin heterobarrier is a heterostructure with the so-called type-II (or staggered) band alignment. In the case of a single heterojunction with the staggered band alignment, ${ }^{13}$ a special type of an interface exciton should result (Fig. 2). The electron part of this heteroexciton is located in semiconductor $B$, in which the conduction band $(\mathrm{CB})$ is below the $\mathrm{CB}$ in semiconductor $A$, and the hole part is placed mainly in semiconductor $A$. In the case of very high levels of exciton pumping in such a heterojunction we can imagine a certain interface electronhole liquid (heteroexciton liquid) concentrated along the heterojunction interface with two separated current directions on different sides of the heterojunction

On the basis of the heterojunction with the staggered band alignment shown in Fig. 2 two types of split heteroexcitons could be organized. They are demonstrated in Fig. 3; a a)
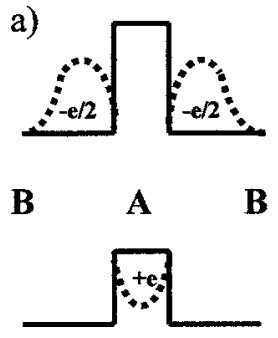

b)
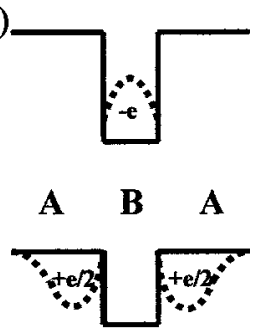

FIG. 3. Two types of split heteroexcitons on the basis of a heterostructure with a staggered band alignment: (a) the model of a split $e$ heteroexciton and (b) the model of a split $h$ heteroexciton. 
split $e$ heteroexciton in Fig. 3(a) and a split $h$ heteroexciton in Fig. 3(b). In contrast to the heteroexcitons formed on the basis of a single heterojunction (Fig. 2), both of the aboveintroduced types of split heteroexcitons are characterized by the S-AS splitting of the ground quantization state. In fact, they are two-level local centers having a two-dimensional (2D) mobility in the heterojunction $x y$ plane. Such excitons could be arbitrarily shifted by inhomogeneous electric fields (for example, created by an ordered system of microgates). The type-II band alignment, which is necessary to obtain both split $e$ heteroexcitons and split $h$ heteroexcitons, is not a unique phenomenon in the heterostructure world. Such alignments take place both in the case of lattice-matched (isomorphic) heterostructures and in the case of strained ones. Both direct-band and multivalley semiconductors could play a role of the electron counterpart (the semiconductor $B$ in Figs. 2 and 3) for the above-considered heterostructures with the staggered alignment. Such heterostructures can be grown on all of the basic types of semiconductor substrates. Let us list several such structures.

(1) GaAs substrates:

The type-II band alignment structures can be grown on the basis of $\mathrm{Al}_{X} \mathrm{Ga}_{1-X} \mathrm{As} / \mathrm{In}_{0.49} \mathrm{Ga}_{0.51} \mathrm{P}, \mathrm{Al}_{X} \mathrm{Ga}_{1-X} \mathrm{As} /$ $\mathrm{In}_{0.48} \mathrm{Al}_{0.52} \mathrm{P}$, and (in the general case) $\mathrm{Al}_{X} \mathrm{Ga}_{1-X} \mathrm{As} /$ $\left(\operatorname{In}_{0.48} \mathrm{Al}_{0.52}\right)_{Y}\left(\operatorname{In}_{0.49} \mathrm{Ga}_{0.51}\right)_{1-Y} \mathrm{P}$ heterojunctions. In the first case staggered structures appear ${ }^{14,15}$ at $X>X_{1}$ $\cong 0.12$. An increase in $X-X_{1}$ leads not only to an increase in a discontinuity $\delta \varepsilon_{C}$ for the conduction bands, but to a simultaneous decrease in a discontinuity $\delta \varepsilon_{V}$ for the valence bands (see Fig. 2). As a result of this decrease the second critical point $X=X_{2}$ appears. At this point the discontinuity $\delta \varepsilon_{V}$ changes its sign, and the band alignment becomes straddling (type I) at $X>X_{2}$. The very crude estimation of $X_{2}$ (using data from the review article Ref. 16) gives $X_{2} \cong 0.4$.

In the case of $\mathrm{Al}_{X} \mathrm{Ga}_{1-X} \mathrm{As} / \mathrm{In}_{0.48} \mathrm{Al}_{0.52} \mathrm{P}$ heterojunctions staggered band alignment structures should take place in the much wider interval of values of $X$. This interval extends probably from some value $X_{1}<0.1$ up to $X=1$ since $\operatorname{In}_{0.48} \mathrm{Al}_{0.52} \mathrm{P}$ is a multivalley semiconductor with the direct band gap ${ }^{16} \sim 2.7 \mathrm{eV}$ and with the $X$-valley indirect band gap $\sim 2.35 \mathrm{eV}$. Therefore, such a heterojunction forms a staggered band alignment both in the case of the direct band gap $\mathrm{Al}_{X} \mathrm{Ga}_{1-X} \mathrm{As}(X<0.45)$ and in the opposite case with $X>0.45$.

(2) InP substrates:

Heterostructures with the type-II (staggered) band alignment lattice matched to InP substrates are still more varied than the previous group. This variety includes first of all the classic $\operatorname{In}_{0.52} \mathrm{Al}_{0.48} \mathrm{As} / \mathrm{InP}$ heterojunction and also additionally ${ }^{17}$ all of the $\operatorname{In}_{0.53-0.02 X} \mathrm{Ga}_{0.47-0.98 X} \mathrm{Al}_{X} \mathrm{As} / \mathrm{InP}$ heterojunctions for $0.48>X>0.22$. Next, this variety includes $\mathrm{GaAs}_{0.5} \mathrm{Sb}_{0.5} / \mathrm{InP}$ heterojunction and the very interesting almost symmetric $\mathrm{GaAs}_{0.5} \mathrm{Sb}_{0.5} / \mathrm{In}_{0.53}$ $\mathrm{Al}_{0.47} \mathrm{As}$ heterojunction. All of the above-listed heterojunctions lattice matched to InP substrates are directband structures. This list could be enlarged by a $\mathrm{AlAs}_{0.56} \mathrm{Sb}_{0.44} / \mathrm{InP}$ heterojunction ${ }^{18-20}$ including as a component the widest band-gap semiconductor $\left(\mathrm{AlAs}_{0.56} \mathrm{Sb}_{0.44}\right)$ lattice matched to InP substrates.

(3) InAs and GaSb substrates:

They allow one to grow a large number of structures with the staggering band alignment. The most typical and known examples are AlSb/InAs heterojunctions and $\mathrm{Al}_{1-X} \mathrm{Ga}_{X} \mathrm{Sb} / \mathrm{InAs}$ heterojunctions for comparatively small values of $X$. Note that the classification including only two types of the band lineup is incomplete in the case of heterostructures grown on these substrates. ${ }^{16}$ The straddling (type I) and staggered (type II) band alignments should be enlarged by the broken-gap (type III) band alignment. The complete collection of the structures grown on these substrates deserves a separate detailed consideration.

(4) Si-based heterostructures:

$\mathrm{Si}_{1-X} \mathrm{Ge}_{X} / \mathrm{Si}$ heterojunctions grown on virtual $\mathrm{Si}_{1-X} \mathrm{Ge}_{X}$ substrates and mentioned above in Sec. III have ${ }^{6}$ the staggered alignment for all the values of $X$. The discontinuity in the conduction band between $\Delta_{2}$ valleys in the tensile-strained $\mathrm{Si}$ and all the valleys in the unstrained $\mathrm{Si}_{1-X} \mathrm{Ge}_{X}$ substrate exceeds $\sim 0.2 \mathrm{eV}$ for $X \geqslant 0.4$ and reaches $\sim 0.5 \mathrm{eV}$ for $X \approx 0.75-0.8$. The discontinuity in the valence band between the light-hole (LH) band, which is the highest in the tensile-strained $\mathrm{Si}$, and the $\mathrm{LH}$ and heavy hole $(\mathrm{HH})$ bands in the substrate is noticeably smaller, $\sim 0.1-0.15 \mathrm{eV}$, but the density of hole states in the substrate material is substantially larger. Therefore, we believe that in the case of the comparatively thin $\mathrm{Si}_{1-X} \mathrm{Ge}_{X}$ layer embedded into the tensilestrained $\mathrm{Si}$ region, the exciton hole (in the $\mathrm{HH}$ form) localizes mainly into this layer and the exciton electron (in the $\Delta_{2}$ form) shifts in $\mathrm{Si}$ on both sides of this layer.

The consideration of the split heteroexciton in the form of the bound electron and hole means that we use the Wannier-Mott approach, ${ }^{21-23}$ in accordance with which the size of this formation substantially exceeds the lattice constant. Below we consider only the split $e$ heteroexciton [Fig. 3(a)] from the two possible versions shown in Fig. 3 since this one is genetically connected with the donor center considered above. In particular, such a situation corresponds to a thin $\mathrm{In}_{0.52} \mathrm{Al}_{0.48} \mathrm{As}$ layer grown in the InP medium, to a thin $\mathrm{GaAs}_{0.5} \mathrm{Sb}_{0.5}$ layer grown in the $\operatorname{In}_{0.53} \mathrm{Al}_{0.47} \mathrm{As}$ medium or in the same InP medium, and also to the thin $\mathrm{Si}_{1-X} \mathrm{Ge}_{X}$ layer grown in the tensile-strained $\mathrm{Si}$ medium, which is grown for its part on the virtual $\langle 100\rangle \mathrm{Si}_{1-X} \mathrm{Ge}_{X}$ substrate.

The thin heterostructural barrier for electrons in the conduction band can be described by a $\delta$-shaped potential $U_{0} \delta(z)$ as above, and the multiplier $U_{0}$ is the single parameter of this description. The same heterostructural layer also serves as the quantum well in the valence band, which localizes a hole. We assume that this well is sufficiently deep and the $\mathrm{HH}$ effective mass is sufficiently large to arrange a sufficiently deep position of the hole ground quantization energy level, to confine the corresponding hole wave function within the layer, and to neglect its penetration outside. The quantized hole has a 2D dispersion relation, which is assumed to be known. In an isotropic and parabolic approxi- 
mation this relation is described by the hole effective mass $m_{P}$. We assume that not only this mass is known but we know also the energy of the hole ground quantization level $-\varepsilon_{G}$ counted from the bottom of the conduction band outside the heterostructural layer. This means that we know the energy gap $\varepsilon_{G}$ between the bottom of the conduction band in the surrounding semiconductor medium and the hole ground quantization energy level. The exciton wave function $\Psi\left(\mathbf{r}_{N}, z_{N} ; \mathbf{r}_{P}\right)$ is a function of five variables: three electron coordinates $\left(\mathbf{r}_{N}=\left\{x_{N}, y_{N}\right\}\right.$ and $\left.z_{N}\right)$ and two hole coordinates $\left(\mathbf{r}_{P}=\left\{x_{P}, y_{P}\right\}\right)$. The Hamiltonian of such a heteroexciton can be written in the form

$$
\begin{aligned}
\hat{H}= & \left(\hat{p}_{N}^{2}+\hat{p}_{Z}^{2}\right) / 2 m_{N}+\hat{p}_{P}^{2} / 2 m_{P}-e^{2} / \kappa_{D}\left(r^{2}+z^{2}\right)^{1 / 2}+\varepsilon_{G} \\
= & -\left(\hbar^{2} / 2 M\right) \partial^{2} / \partial \mathbf{R}^{2}-\left(\hbar^{2} / 2 \mu_{N} M\right) \partial^{2} / \partial z^{2} \\
& -\left(\hbar^{2} / 2 \mu_{N} \mu_{P} M\right) \partial^{2} / \partial \mathbf{r}^{2}-e^{2} / \kappa_{D}\left(r^{2}+z^{2}\right)^{1 / 2}+\varepsilon_{G},
\end{aligned}
$$

where $\quad \hat{p}_{N, P}=-i \hbar \partial / \partial \mathbf{r}_{N, P}, \hat{p}_{Z}=-i \hbar \partial / \partial z, M=m_{N}+m_{P}, \mu_{N, P}$ $=m_{N, P} / M, \quad \mathbf{R}=\mu_{N} \mathbf{r}_{N}+\mu_{P} \mathbf{r}_{P}, \quad \mathbf{r}=\mathbf{r}_{N}-\mathbf{r}_{P}, \quad$ and $z=z_{N} . \quad$ The Hamiltonian (25) allows us to separate the variables in the Schrödinger equation corresponding to this Hamiltonian and introduce the multiplicative function

$$
\Psi=\Phi(\mathbf{R}) \psi(\mathbf{r}, z),
$$

where $\Phi(\mathbf{R})$ describes a free motion of the 2D heteroexciton with the mass $M$ and $\psi(\mathbf{r}, z)$ describes a three-dimensional (3D) motion of the electron bound by the hole around the exciton mass center. This $3 \mathrm{D}$ motion is described by two effective masses: $m_{\perp}=m_{N} m_{P} /\left(m_{N}+m_{P}\right)$ and $m_{Z}=m_{N}>m_{\perp}$. Since as a rule $m_{N}$ is substantially smaller than $m_{P}$, which is close to the $\mathrm{HH}$ effective mass, this additional anisotropy is not very large. Therefore, the above-calculated estimation of the S-AS splitting of the ground state donor level [see Eq. (10)] can be satisfactory.

In the most interesting case of a $\mathrm{Si}_{1-X} \mathrm{Ge}_{X} / \mathrm{Si}$ heterostructure, the quantized hole confined in the hole $\mathrm{Si}_{1-X} \mathrm{Ge}_{X}$ quantum well binds an anisotropic $\Delta_{2}$ electron located mainly in Si. Then a free motion of the $2 \mathrm{D}$ exciton is described by effective mass $M=m_{N \perp}+m_{P}$ and quantization of the bound electron is defined by two effective masses,

$$
m_{\perp}=m_{N \perp} m_{P} /\left(m_{N \perp}+m_{P}\right), \quad m_{Z}=m_{N Z},
$$

where $m_{N \perp}$ and $m_{N Z}$ are the effective masses of an anisotropic $\Delta_{2}$ electron. We can see that the $\Delta_{2}$ electron trapped by the heteroexciton is somewhat more anisotropic than the same electron trapped by the donor center since $m_{\perp}<m_{N \perp}$ but this effect is not very large because $m_{N \perp}$ can be noticeably smaller than $m_{P}$.

Above we restricted our description of a quantized hole dispersion relation by the single parameter $m_{P}$. First, we have neglected the well-known nonparabolic behavior of subbands of size-quantized holes. Second, we have neglected their noticeable anisotropy in the $x y$ plane. The first and the second become very substantial ${ }^{24-27}$ if $k_{p} w \geqslant 1$ where $k_{p}$ is a wave vector of a quantized hole. We believe that these effects are negligible if $r_{B} \gg w$ [see Eq. (1)] where the electron Bohr radius $r_{B}$ determines the exciton size. We should consider sufficiently thin heterostructural layers. Nevertheless, these layers should reliably provide the $\mathrm{HH}$ quantization.

\section{CONCLUDING REMARKS}

We have considered two methods of formation of twolevel quantum systems. Both methods are based on growing very thin heterostructural layers $(w \leqslant 1 \mathrm{~nm})$ in a homogeneous semiconductor medium. In the first case, a positively charged donor center binding an electron is embedded in the layer that is a potential barrier for electrons and the electron wave function is located symmetrically on different sides of this barrier. Both these electron halves are combined by a weak tunnel connection through the barrier. A disadvantage of this method could be caused by insufficient symmetry of both the thin heterostructural layer itself and a position of the donor center inside the layer. The other disadvantage could be connected with the necessity to align positions of the above-mentioned donor centers and the controlling microgates.

The second method consists of replacing the donor center by a hole binding an electron and forming a heteroexciton together with it. Such a split heteroexciton is localized around the heterostructural layer only if the staggered band alignment takes place with the formation of a thin potential heterobarrier for the electron and a quantum well confining the hole. This exciton could be shifted along the heterostructural layer with the help of a controlling inhomogeneous electric field ${ }^{28}$ and placed in the desirable position. The spatial symmetrization of the exciton also looks simpler than the electron symmetrization in a structure with a localized donor center.

Split excitons can be obtained by connecting injected free electrons and holes in the distinctive $p^{+}-i-n^{+}$structure where a remote $n^{+}$contact injects electrons directly in semiconductor $B$ and a remote $p^{+}$contact injects holes in the hole quantum well $A$. The success of such a measure depends critically on the lifetime of the split heteroexciton, which should be maximal and should repeatedly exceed a time of formation of this pair from the injected free quasiparticles. Of course, the thin heterostructural layer containing the hole quantum well and the electron tunnel heterobarrier should be maximally homogeneous.

\section{ACKNOWLEDGMENTS}

The authors thank participants of the IQS seminar at Michigan State University (MSU) for the fruitful discussion. This work has been supported by Institute of Quantum Sciences (IQS) at MSU and the Solid State Electronics Laboratory at the University of Michigan.

${ }^{1}$ L. D. Landau and E. M. Lifshitz, Quantum Mechanics: Nonrelativistic Theory (Butterworth-Heinemann, Oxford, 2000).

${ }^{2}$ A. Messiah, Quantum Mechanics (North Holland, Amsterdam, 1961).

${ }^{3}$ S. Flügge, Practical Quantum Mechanics I (Springer, Berlin, 1971).

${ }^{4}$ M. Friesen, P. Rugheimer, D. E. Savage, M. G. Lagally, D. W. van der Weide, R. Joint, and M. A. Eriksson, Phys. Rev. B 67, 121301 (2003).

${ }^{5}$ R. Vrijen, E. Yablonovitch, K. Wang, H. W. Jiang, A. Balandin, V. Roychowdhury, T. Mor, and D. DiVincenzo, Phys. Rev. A 62, 012306 (2000). ${ }^{6}$ F. Schäffler, Semicond. Sci. Technol. 12, 1515 (1997).

${ }^{7}$ M. Holzmann, D. Többen, G. Abstreiter, and F. Schäffler, J. Appl. Phys. 76, 3917 (1994)

${ }^{8}$ M. Holzmann, D. Többen, G. Abstreiter, M. Wendel, H. Lorenz, J. P. Kotthhaus, and F. Schäffler, Appl. Phys. Lett. 66, 833 (1995).

${ }^{9}$ C. Kittel and A. Mitchell, Phys. Rev. 96, 1488 (1954). 
${ }^{10}$ M. A. Lampert, Phys. Rev. 97, 352 (1955).

${ }^{11}$ W. Kohn and J. M. Luttinger, Phys. Rev. 98, 915 (1955).

${ }^{12}$ R. A. Faulkner, Phys. Rev. 184, 713 (1968).

${ }^{13}$ H. Kroemer and G. Griffiths, IEEE Electron Device Lett. 4, 20 (1983).

${ }^{14}$ K.-S. Kim, Y.-H. Cho, B.-D. Choe, W. G. Jeong, and H. Lim, Appl. Phys. Lett. 67, 1718 (1995).

${ }^{15}$ I.-J. Kim, Y.-H. Cho, K.-S. Kim, B.-D. Choe, and H. Lim, Appl. Phys. Lett. 68, 3488 (1996).

${ }^{16}$ I. Vurgaftman, J. R. Meyer, and L. R. Ram-Mohan, J. Appl. Phys. 89, 5815 (2001).

${ }^{17}$ J. Böhrer, A. Krost, and D. B. Bimberg, Appl. Phys. Lett. 63, 1918 (1993).

${ }^{18}$ S. Muto and T. Inata, Semicond. Sci. Technol. 9, 1157 (1994).

${ }^{19}$ D. C. Dumka, G. Sueva, H. Heir, O. A. Aina, and I. Adelsida, IEEE Electron Device Lett. 22, 5 (2001).
${ }^{20}$ A. Neogi, H. Yoshida, T. Mosume, N. Georgiev, and O. Wada, IEEE J. Sel. Top. Quantum Electron. 7, 710 (2001).

${ }^{21}$ C. Kittel, Introduction to Solid State Physics (Wiley, New York, 1986), Chap. 11.

${ }^{22}$ A. I. Ansel'm, Introduction to Theory of Semiconductors (Nauka, Moscow, 1978), Chap. 7.

${ }^{23}$ O. Madelung, Festcörpertheorie (Springer, Berlin, 1972), Chap. 7.

${ }^{24}$ L. C. Andreani, A. Pasquarello, and F. Bassani, Phys. Rev. B 36, 5887 (1987).

${ }^{25}$ R. Winkler and U. Rössler, Phys. Rev. B 48, 8918 (1993).

${ }^{26}$ Z. N. Sokolova, V. B. Khalfin, and Al. L. Efros, Fiz. Tekh. Poluprovodn. (S.-Peterburg) 22, 2124 (1988) [Sov. Phys. Semicond. 22, 1342 (1988)].

${ }^{27}$ Al. L. Efros, Fiz. Tekh. Poluprovodn. (S.-Peterburg) 20, 1281 (1986) [Sov. Phys. Semicond. 20, 808 (1986)].

${ }^{28}$ Z. S. Gribnikov and E. I. Rashba, Zh. Tekh. Fiz. 28, 1948 (1958). 